\title{
A Component Mutation of virB Virulence Affects Lysogenization bY Phage P22
}

Several genes of the temperate Salmonella phage P22 have been implicated in the process of lysogenization. A mutation in any of three adjacent regulatory genes, $c_{1,2} c$, or $c_{3}$, confers a clear plaque morphology and the inability to lysogenize (5). The $c_{1}$ and $c_{3}$ gene products are required for the establishment but not the maintenance of lysogeny. On the other hand, the $c_{2}$ gene product is required for maintenance of lysogeny and has the properties of a repressor $(7)$. The function of another regulatory gene, $m n t$, unlinked to the clear region, is also required for the maintenance of lysogeny (4). Several additional P22 genes must be expressed for the integration process itsolf to occur. The int gene appears to code for a site-specific recombination enzyme required for both integration and excision $(8,10)$. The products of genes 12 and 18 have dual functions in promoting both phage DNA synthesis and integration (6). The erf gene product is required for phage DNA synthesis, phage recombination, and integration in a $\operatorname{Rec}^{-}$but not a $R e c^{+}$host (1).

In previous communications we have describod a virulent mutant of phage P22, vir $B-3$, which can productively infect a $\mathrm{P} 22$ lysogen and is unable to lysogenize a sensitive host (2, 3). P2Q virB-3 consists of two mutations, both of which are required for virulence: $K 5$ which maps in the $c_{2}$ gene, and $V x$ which maps between genes $c_{2}$ and $c_{3}$. P22K5 makes large clear plaques and behaves as a typical $c_{2}$ mutant in lysogenization tests: it comploments for lysogeny in mixed infections with $c_{1}$ and $c_{3}$ mutants but not with $c_{2}$ mutants $(\mathscr{Q}, 5)$. In contrast to P2OK5, PQ2Vx makes small turbid plaques on a sensitive host and is not mutant in any of the clear genos. Nevertheless, preliminary evidence suggested that cells infected by $P 22 V x$ fail to give rise to lysogenic progeny. The results presented in this communication confirm these findings and indicate that the $V x$ mutation is responsible for a defect in the maintenance of lysogeny.

To determine if the $V x$ mutation affects lysogenization, the segregation pattern of $P \mathscr{P V} x$-infected sensitive cells was compared to that of cells infected by wild-type P22. Log phase Salmonella typhimurium strain 18 cells were grown in supplemented M9 medium (9) to a cell concentration of $10^{8} / \mathrm{ml}$ and infected at a multiplicity of 20 at $33^{\circ} \mathrm{C}$ to maximize the frequency of lysogenization (10). After a 5-min adsorption period, the cultures were diluted into growth medium containing antiphage serum. At intervals, aliquots were plated on EMB galactose agar (5) and incubated at $25^{\circ} \mathrm{C}$ for $48 \mathrm{hr}$ for determination of the frequencies of sensitive cells, cells carrying phage genomes and total cells.

In the control infection with wild-type $c^{+}$ phage about $95 \%$ of the cells survived as phage carriers. The surviving cells began dividing after a lag of about one generation, and thereafter the total number of cells increased logarithmically (Fig. 1A). Segregation of phage carriers and non-phage carriers occurred soon after cell division resumed. After a few generations segregation ceased, and the ratio of phage carriers to total cells remained constant. These data are interpreted as suggested by Smith and Levine (1967). After an early period of segregation of intracellular phage genomes, prophage integration has occurred, because the capacity to produce phage is perpetuated synchronously with cell growth.

Infection of the senstive host by P22Vx also resulted in nearly $100 \%$ survival. The surviving cells carried phage genomes in the initial stages of infection. As in the case of the wild-typo infection, cell division resumed after a lag of about one generation (Fig. 1B). However, after a few generations of logarithmic cell growth, the number of phage carriers remained constant. The ratio of phage carriers to total cells continued to decreased with each division, indicating the absence of stable lysogeny. This segregation pattern is similar to that described for integration-deficient int mutants (10) and for mutants in genes 12 and 18 which cannot integrate (6).

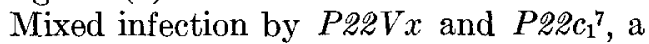
phage defective in the establishment but not the maintenance of lysogeny $(5,7)$, resulted 

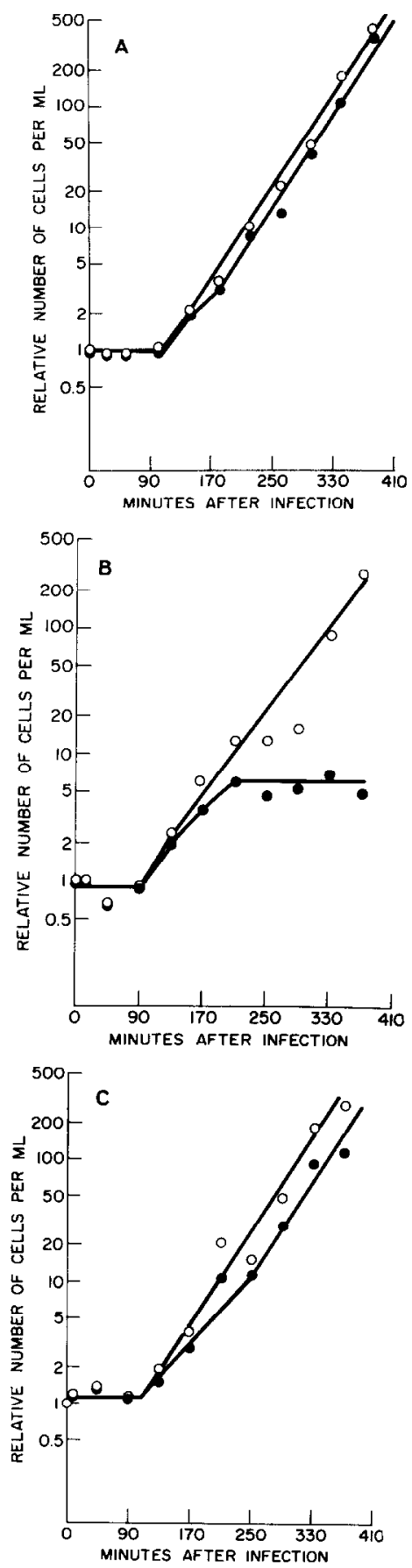

FIG. 1. Segregation of sensitive cells and cells carrying phage genomes after infection at $33^{\circ} \mathrm{C}$ with: A, wild-type phage at m.o.i. of 20 ; B, $P 2 \mathscr{Q} V x$ at m.o.i. of 20 ; and $\mathrm{C}, P 2 Q 2 c_{1}{ }^{7}$ and $P 2 Q V x$ at m.o.i. of 10 each. Cell numbers are relative to the initial number of infected cells. - $\mathrm{O}-\mathrm{O}$, total number of cells; - - number of cells carrying phage genomes. in complementation for lysogeny (Fig. 1C). The segregation pattern at $33^{\circ} \mathrm{C}$ resembled that of the wild-type $c^{+}$control infection. However, lysogens carried only $c_{1}$ prophage. Double lysogens were not detected. Stable lysogens for $V x$ prophage have not been observed, suggesting that the inability of PQ2VX to lysogenize is a consequence of a defect in maintenance of prophage.

The maintenance defect of $P \mathscr{Q} Q V x$ may be due to expression of gene functions ordinarily repressed in the lysogenic state. Although the specific genes regulated at the $V x$ site are not known, P22Vx in the presence of repressor can supply a function(s) in trans which is required for phage DNA synthesis (3). It is conceivable that the int and xis genes are part of an operon controlled at the $V x$ site. Since the int and $x i$ functions are the two gene products known to be required for proprophage excision, synthesis of these would favor excision over integration. Alternatively, the inability of the $V x$ genome to be maintained as prophage may be independent of translation. That is, constitutive transcription itself might interfere with stable integration of the $V x$ genome.

\section{REFERENCES}

1. Botstein, D., and MАtz, M., J. Mol. Biol. 54, 417-440 (1970).

2. Bronson, M. J., and Levine, M., J. Virol. 5, 559-568 (1971).

3. Bronson, M. J., and Levine, M., Virology 47, 644-655 (1972).

4. Govgh, M., J. Virol. 2, 992-998 (1968).

5. Levine, M., Virology 13, 493-499 (1957).

6. Levine, M., and Schotт, C., J. Mol. Biol. 62, 53-64 (1971).

7. Levins, M., and Smith, H. O., Science 146, 1581-1582 (1964).

8. Sмгтн, H. O., Virology 34, 203-223 (1968).

9. Smith, H. O., and Levine, M., Proc. Nat. Acad. Sci. USA 52, 356-363 (1964).

10. Sмiт'H, H. O., and Levine, M., Virology 31, 207-216 (1967).

Morley J. Bronson ${ }^{1}$ Mrron Levine

Department of Human Genetics

University of Michigan

Ann Arbor, Michigan 48104

Accepted October 30,1972

${ }_{1}$ Present address: Department of Biological Sciences, Stanford University, Stanford, California 94305 . 